\title{
Migraine in recurrent depression: case-control study
}

Zainab Samaan, Anne Farmer, Nick Craddock, Lisa Jones, Ania Korszun, Mike Owen and Peter McGuffin

\section{Background}

An association between depression and headache is well established, but the specificity to migraine is unclear.

\section{Aims}

To investigate the specificity of the association of depression and migraine.

\section{Method}

People with recurrent depression $(n=1259)$ were compared with psychiatrically healthy controls $(n=851)$ to investigate headache defined according to International Headache Society criteria in each group.

\section{Results}

All headache types were more prevalent in the case group than in the controls. However, the strongest association was between depression and migraine with aura $(\mathrm{OR}=5.6)$. Among participants with recurrent headaches, migraine with aura (but not other forms of headache) was highly significantly associated with depression.

\section{Conclusions}

The data suggest that not only is there a general relationship between headache and depression but also that among people with recurrent headache there is a specific association between depression and migraine with aura. The association is likely to be explained by overlapping aetiological risk factors.

\section{Declaration of interest}

M.O. and N.C. have acted as consultants to GlaxoSmithKline (GSK) and have received honoraria for academic talks from Eli Lilly, AstraZeneca and GSK. A.K. has received research grants from GSK and Sanofi-Synthelabo and has received honoraria from Eli Lilly. A.F. has received honoraria for presentations and chairing meetings from Eli Lilly, GSK and Wyeth, and has acted as a consultant for GSK. P.M. has received honoraria from Eli Lilly and GSK, and has acted as a consultant in the recent past for GSK and AstraZeneca.
Migraine and depression have long been noted to co-occur and most studies suggest that the frequency of this phenomenon is greater than can be explained by chance. ${ }^{1}$ However, there has also been long uncertainty about the specificity of the association between the two disorders, in that other forms of headache are also frequently associated with depression. ${ }^{2}$ A recent review of studies carried out since the introduction of explicit and widely accepted diagnostic criteria for migraine and other forms of headache suggested that the specificity of the migraine-depression association remains unclear, and its authors concluded that no difference had been demonstrated between migraine patients and people with tension headache in terms of prevalence of psychopathology. ${ }^{2}$ However, nearly all of the evidence on the relationship between migraine and depression is from studies on community-drawn samples or clinic-based samples of patients with headache ${ }^{2}$ rather than people with depression. Hence, we have set out to examine the strength and specificity of the relationship between depression and migraine on a large sample of people with recurrent depression, comparing them with a psychiatrically healthy control group.

\section{Method}

\section{Study population}

The samples were collected primarily to participate in casecontrol genetic association studies. All participants were recruited in the UK. People who had experienced two or more episodes of depression were recruited from psychiatric clinics and hospitals $(26 \%)$, general practice $(46 \%)$ and through self-help groups and media advertisement (28\%) during 2001-4. They were informed that the study was investigating the genetic bases of recurrent depression as well as its comorbidity with medical illnesses such as migraine. Potential participants were included if they were at least 18 years old and of White ethnic background, and were excluded if they had injecting drug dependence, depression occurring only in relation to substance misuse or medical illness, or a personal or family history of mania or mood-incongruent psychotic symptoms.

The control group was selected from among participants who had originally been recruited to the Genetic and Environmental Nature of Emotional States in Siblings (GENESiS) study. ${ }^{3}$ This study recruited 34371 individuals through general practices in England and Wales. Index participants were individuals registered with these practices in the age range 18-55 years who constituted the lowest $20 \%$ of the distribution on the Sham Composite Index of liability to depression and anxiety. ${ }^{3}$ Those who responded positively to a postal invitation to participate in the current study and who had returned signed consent forms were then contacted by telephone, and the Past History Schedule was administered by trained research assistants (graduate psychologists). ${ }^{4}$ Only those with no personal current or past psychiatric disorder and no family history of psychiatric illness in a first-degree relative were included in the study.

All participants gave written informed consent and the protocol was approved by the local ethics committees of the participating institutions.

\section{Diagnostic instruments}

All participants in the depression group were given a face-to-face semi-structured diagnostic interview by trained interviewers, the Schedule for Clinical Assessment in Neuropsychiatry (SCAN), ${ }^{5}$ to establish the diagnosis of recurrent depression with symptoms rated for the worst and second worst episodes of depression. The SCAN interview was scored using the CATEGO5 ${ }^{6}$ program to 
assign ICD-10 and DSM-IV diagnoses. ${ }^{7,8}$ Recurrent depression was defined as a lifetime diagnosis of two or more episodes of illness fulfilling ICD-10 and/or DSM-IV criteria for major depression of at least moderate severity with clear inter-episode recovery or remission of at least 2 months duration. We also used the Beck Depression Inventory ${ }^{9}$ (BDI) to assess depressive symptoms over the past week for both cases and controls.

\section{Structured Migraine Interview}

For the lifetime diagnosis of migraine, a short, structured questionnaire, the Structured Migraine Interview (SMI), was designed using International Headache Society criteria ${ }^{10}$ and administered by telephone by research assistants trained in its use. Detailed questions were asked about the presence of headache, its severity, frequency, duration, site, character and aura, and associated symptoms. The interview consisted of ten questions to yield mutually exclusive categories of headache. The categories assumed a ranking order of severity, with migraine with aura considered to be the most severe form, followed by migraine without aura, probable migraine, other headache and, finally, no recurrent headache. The criteria for diagnosing each category are shown in the Appendix. The responses to the interview were entered into a diagnostic scoring program generated in Stata version 7 on Windows based on the International Headache Society criteria. ${ }^{8}$

The validating characteristics of the SMI were determined by comparing the headache diagnosis derived from this questionnaire using the scoring program with the clinical diagnosis of a consultant neurologist in 41 patients attending the London Migraine Clinic. In addition, an examination of the test-retest reliability of the SMI-derived diagnoses over a mean of 3 years was performed for 20 participants.

\section{Statistical analysis}

Data were entered into the Statistical Package for the Social Sciences (SPSS) version 12 for Windows. Group comparisons of the lifetime prevalence of migraine and other headaches were performed using simple contingency table analyses and logistic regression. In the logistic regression analysis, significance was assessed using the Wald statistic.

\section{Results}

There were 1259 persons with recurrent depression recruited to the genetic case-control study, all of whom fulfilled ICD-10 and/or DSM-IV criteria for recurrent major depressive disorder. The mean age of this group was 47.3 years $($ s.d. $=12.5$, range 19 $85)$. The control group $(n=851)$ had a similar mean age of 47.9 years (s.d.=9.1, range 20-69). The majority of participants were women in both the case and control groups; however, there were significantly more women in the case group than in the control group ( $70 \%$ v. 56\%; Pearson $\chi^{2}=47.4$, d.f. $=1, P<0.0001$ ). All the participants were White and lived in the UK. More than half of those in the case group had post-secondary school education compared with $44 \%$ of the control group; however, the majority of the control group were in active employment (81\%) compared with only $46 \%$ of the case group. The case group had a slightly higher mean body mass index (BMI; $26.8 \mathrm{~kg} / \mathrm{m}^{2}$ ) than the control group $\left(25.2 \mathrm{~kg} / \mathrm{m}^{2}\right)$.

\section{Validity and reliability of the SMI}

Compared with a consultant neurologist's diagnoses in 41 patients with headache attending the London Migraine Clinic, the
SMI-derived diagnoses had an excellent specificity (1.00) and modest sensitivity (0.66). In addition, for 20 of the participants with depression, the test-retest reliability of the 'lifetime ever' diagnosis of migraine was determined over a mean interval of 3 years (range 1-4). Spearman's correlation coefficient (rho) was $0.8(P<0.001)$.

\section{Case-control comparisons}

Table 1 shows the distribution of various headache and migraine categories in both study groups using the SMI. All headache types were more prevalent in the case group $(61.0 \%)$ than in the control group $(31.6 \%)$. Broadly defined migraine (migraine with and without aura, and probable migraine) occurred more frequently in the case group ( $32.4 \%$ v. $12.8 \%$; Pearson $\chi^{2}=105.4$, d.f. $=1$, $P<0.0001)$.

Although the mean ages for cases and controls were similar, there were more older adults in the case group than in the control group. To investigate any significant difference in migraine rates in different age groups, the sample was divided into those under 60 years and those aged 60 years or older. No difference was detected in lifetime ever migraine rates for all participants 60 years old or over when interviewed, compared with those under 60 years old $\left(\chi^{2}=0.2\right.$, d.f. $\left.=1, P=0.69\right)$. Similarly, the lifetime ever reporting of migraine in the younger people with depression when interviewed was similar to that in the case group who were 60 years old or over $\left(\chi^{2}=3.2\right.$, d.f. $\left.=1, P=0.08\right)$.

Broadly defined migraine was twice as common in women $(30 \%)$ than in men $(15 \%)$ in the whole sample (Pearson $\chi^{2}=60.3$, d.f. $=1, P<0.0001$ ), and also occurred significantly more frequently in women in the case group compared with women in the control group $\left(\chi^{2}=53\right.$, d.f. $\left.=1, P<0.0001\right)$. The prevalence of migraine and other headaches was estimated separately for each gender to examine for the effect of female overrepresentation in the case group. Both men and women in the case group had more than twice the rate of broadly defined migraine compared with controls (Table 2). The difference between cases and controls segregated by gender in the rates of each headache category was statistically significant (female: Pearson $\chi^{2}=98.1$, d.f. $=5$, $P<0.0001$; male: Pearson $\chi^{2}=66.04$, d.f. $=5, P<0.0001$ ).

To investigate the significance of gender and headache type in migraine comorbidity with depression we performed a logistic regression analysis with case/control assigned as the dependent variable and headache types, BMI, education and employment plus gender as the explanatory variables. Table 3 shows the results of logistic regression analysis. All headache types, migrainous and non-migrainous, were positively associated with depression. The risk of developing migraine with aura in participants with depression was higher than the risk of other headache disorders. In addition, female gender was significantly associated with depression. Education level, employment and BMI were not significantly associated with depression. Non-migrainous

Table 1 Migraine and other headache distribution in cases and controls

\begin{tabular}{|lccc|}
\hline & $\begin{array}{c}\text { Cases } \\
n(\%)\end{array}$ & $\begin{array}{c}\text { Controls } \\
n(\%)\end{array}$ & $\begin{array}{c}\text { Total } \\
n\end{array}$ \\
\hline Migraine with aura & $122(9.7)$ & $23(2.7)$ & 145 \\
\hline Migraine without aura & $67(5.3)$ & $20(2.4)$ & 87 \\
\hline Probable migraine & $219(17.4)$ & $66(7.8)$ & 285 \\
\hline Non-migraine headache & $360(28.6)$ & $160(18.8)$ & 520 \\
\hline No recurrent headache & $491(39.0)$ & $582(68.3)$ & 1073 \\
\hline Total & $1259(100.0)$ & $851(100.0)$ & 2110 \\
\hline
\end{tabular}




\begin{tabular}{|c|c|c|c|c|c|}
\hline & \multicolumn{2}{|c|}{ Women $(n=1357)$} & \multicolumn{2}{|c|}{ Men $(n=753)$} & \multirow[b]{2}{*}{$\begin{array}{c}\text { Total }(n=2110) \\
n\end{array}$} \\
\hline & $\begin{array}{c}\text { Cases }(n=884) \\
n(\%)\end{array}$ & $\begin{array}{c}\text { Controls }(n=473) \\
n(\%)\end{array}$ & $\begin{array}{c}\text { Cases }(n=375) \\
n(\%)\end{array}$ & $\begin{array}{c}\text { Controls }(n=378) \\
n(\%)\end{array}$ & \\
\hline Migraine with aura & $104(11.8)$ & $18(3.8)$ & $18(4.8)$ & $5(1.3)$ & 145 \\
\hline Migraine without aura & $51(5.8)$ & $16(3.4)$ & $16(4.3)$ & $4(1.1)$ & 87 \\
\hline Probable migraine & $168(19.0)$ & $49(10.4)$ & $51(13.6)$ & $17(4.5)$ & 285 \\
\hline Non-migraine headache & $257(29.1)$ & $100(21.1)$ & $103(27.5)$ & $60(15.9)$ & 520 \\
\hline No recurrent headache & $304(34.4)$ & $290(61.3)$ & $187(49.9)$ & $292(77.2)$ & 1073 \\
\hline
\end{tabular}

\begin{tabular}{|c|c|c|c|c|}
\hline Variables & $\mathrm{OR}(95 \% \mathrm{Cl})$ & Wald $\chi^{2}$ & d.f. & $P$ \\
\hline Migraine with aura & $5.6(3.54-9.0)$ & 53.15 & 1 & $<0.0001$ \\
\hline Migraine without aura & $3.7(2.2-6.14)$ & 24.31 & 1 & $<0.0001$ \\
\hline Probable migraine & $3.6(2.7-5.0)$ & 70.03 & 1 & $<0.0001$ \\
\hline Non-migraine headache & $2.6(2.0-3.2)$ & 67.3 & 1 & $<0.0001$ \\
\hline Female gender & $1.5(1.3-1.8)$ & 18.5 & 1 & $<0.0001$ \\
\hline
\end{tabular}

headache was modestly significantly associated with depression, but there were stronger associations with probable migraine, migraine with aura and - most strikingly - migraine without aura.

In order to test for specificity of the association between migraine and depression we repeated the regression analysis excluding all participants (cases and controls) who did not experience any form of recurrent headache. The odds ratio (OR) for the association of migraine with aura and recurrent depression in this headache group was 2.0 (95\% CI 1.3-3.2; Wald $\chi^{2}=8.8$, d.f. $=1, P=0.003$ ). Other types of headache lost their significant association with depression, suggesting that the association is somewhat specific to migraine with aura. The results were also not significant for gender $(\mathrm{OR}=1.3,95 \%$ CI $1.0-1.6$; $P=0.07)$.

\section{Discussion}

Our results are in keeping with many previous reports indicating a consistent association between migraine, other headaches and depression. Where our study differs from previous investigations is that the findings are based upon a large sample of clinically well-defined cases of recurrent depression compared with carefully screened psychiatrically healthy controls. This enabled us not only to assess the strength and significance of various types of headache with recurrent depression, but also allowed us to focus on those individuals (both cases of depression and controls) who experienced recurrent headaches in order to test for specificity of the association between migraine and depression. We found a highly significant and moderately strong overall effect such that participants with headache who also had recurrent depression were more likely than participants who had never been depressed to report migraine with aura. Interestingly, the strength of the effect as judged by odds ratios was, in ascending order, probable migraine, migraine without aura and migraine with aura - that is, the more 'classic' the pattern of the migraine symptoms, the more strongly they were associated with depression. Indeed, the odds ratio for the migraine with aura association with recurrent depression was 5.6 in all participants and 2.0 for the association between any type of headache and depression. Although high rates of depression have been reported in those experiencing migraine with aura compared with those having other types of migraine, ${ }^{11}$ no previous study has explored the frequency of migraine with aura and other types of migrainous and non-migrainous headache in people selected for having recurrent depression. Furthermore, no previous study has been able to conclusively differentiate whether there is a particular association between migraine and depression as opposed to headaches generally. ${ }^{2}$

The possible reasons for an association between migraine and depression cannot be conclusively decided on the basis of our results. However, some reasonable inferences can be made from the pattern of the findings and in the light of previous studies. Investigations of the temporal relationship between psychiatric and migraine symptoms have tended to find that anxiety symptoms precede those of migraine, ${ }^{12-14}$ which in turn precedes depression. Although this could mean that recurrent migrainous headaches have a causal role in the development of later depressive symptoms, the data are by no means consistent and it is difficult to argue that the recurrent suffering resulting from migrainous headaches should be any more depressogenic than for headaches of other types. Other studies have suggested a bidirectional relationship between migraine and depression, with each disorder increasing the susceptibility for developing the other. However, this relationship, although consistent, remains poorly understood. ${ }^{11}$ Another likely explanation is that there is a genetic-neurodevelopmental relationship between the disorders. Anxiety symptoms often precede those of depression, and twin studies suggest a large overlap between the genes predisposing to symptoms of depression and anxiety. ${ }^{15,16}$ No twin study in adults has examined the genetic overlap between depression and migraine, although Merikangas et al found evidence of transmission of both disorders in families ascertained through depressed probands, but were unable to distinguish formally between environmental and genetic effects. ${ }^{17}$ Depressive disorder, especially that which is recurrent and severe enough to lead to impairment, is substantially heritable. ${ }^{18,19}$ Similarly, there appears to be an important genetic component in migraine, which is not confined to rare Mendelian subtypes. ${ }^{1,20}$ It is well known that antidepressants can be effective in the treatment of migraine, ${ }^{21}$ adding another clue to the shared mechanisms between the two disorders. In addition, in support of the genetic relationship between migraine and depression, several candidate gene studies reported significant association between a C677T polymorphism in the methylenetetrahydrofolate reductase gene and migraine 
and depression. ${ }^{22,23}$ More family and twin studies examining both disorders are sorely needed.

It is also possible that retrospective rating of their headache symptoms by people with recurrent depression could have been influenced by their present mood. Indeed, we did find a small but significant correlation between scores on a self-report measure of present mood, the BDI, and number of symptoms reported on the SMI in cases but not in controls. However, BDI scores only contributed $3 \%$ of the variance in headache symptoms reported in cases and $0.4 \%$ in controls. We can also argue that our case group had a higher mean BMI than the control group, indicating a possible confounder to the association between migraine and being overweight. However, this interpretation is not plausible because evidence from large epidemiological studies found no specific relation between the diagnosis of migraine and BMI. ${ }^{24}$

Other factors that may be considered are the differences in occupational status and education. Migraine has been reported to be more prevalent in lower socio-economic groups. ${ }^{25}$ However, this relationship between migraine and level of income was not consistent in other studies outside the USA. ${ }^{26}$ In addition, being psychiatrically healthy may potentially bias the migraine rates in the control group. This is not the case in our study, since the control group showed a similar migraine rate to that of the general population figures of $11.6 \%$ on average in European countries. ${ }^{27}$ Another possible confounding effect is that migraine has been associated with increased cardiovascular risk factors and with high diastolic blood pressure, ${ }^{28,29}$ and in a previous analysis of the present data-set there was an excess of self-reported hypertension and heart disease in cases of depression compared with controls. ${ }^{30}$ However, reports of an association between migraine and elevated blood pressure are inconsistent, ${ }^{31,32}$ and in our data there was no relationship between migraine and heart disease. There was a small significant correlation between migraine and self-reported hypertension, but this accounted for less than $1 \%$ of the variance.

\section{Limitations}

Our study has certain limitations, the most obvious of which is that neither the cases nor the controls were systematically ascertained. Just under half of the case group (46\%) and all the control group were recruited from general practices in the UK. It can be argued that the different sources of recruitment of study cases could have confounded the results. It is expected that the majority of people with psychiatric disorders seek healthcare service in primary care, secondary care or both, and therefore a selection from all available sources will give a better representation of such individuals. Similarly, about a quarter of the cases were self-referred, reflecting a self-selection bias whereby such individuals might have been more inclined to report headache symptoms than others. However, we did not find a significant difference in migraine rates among cases recruited from different sources (general practice $v$. secondary care: $\chi^{2}=0.5, P=0.49$; general practice $v$. self-referral: $\chi^{2}=0.1, P=0.83$ ). In addition, it is unlikely that a volunteer bias would have led to an excess of participants with migraine rather than any other type of headache among the group with recurrent depression. Nor, indeed, would this be likely to explain the rank order effect in which migraine with aura had a stronger association with depression than migraine without aura, which in turn had a stronger association than probable migraine. Instead, we propose that this argues for a probable aetiological overlap that is likely to reflect the involvement of common neurochemical pathways. Indirect evidence that this may be so comes from observations that tricyclic and monoamine oxidase inhibitor antidepressants have efficacy in both disorders, implicating dysfunction in the serotonergic and adrenergic systems. ${ }^{33,34}$

Another possible limitation is that the clinical categories of migraine were based on a short, structured interview that clearly results in more conservative diagnoses than those of an experienced clinician. None the less, the SMI produced no false positive result compared with the clinician diagnoses and this makes it unlikely that some form of response bias was responsible for our observed migraine-depression association. Overrepresentation of women in the case group might also have confounded the results, since it is expected that migraine is more common in women than in men (rates two to three times higher have been reported). ${ }^{26,35}$ For migraine with aura the female: male ratio was $2: 1$ and for migraine without aura it was $7: 1 .^{36}$ However, when the prevalence of migraine and other headaches was analysed on the basis of gender, female participants with depression had more than twice the rate of migraine compared with psychiatrically healthy women.

\section{Future research}

In conclusion, this study of a large sample of well-defined cases of recurrent depression and a psychiatrically healthy control group shows that among people who experience recurrent headache there is a significant association between migraine and depression. Furthermore, we have confirmed that the association is most striking with 'classic' migraine, particularly migraine with aura, and this, we propose, is most readily interpretable as reflecting shared aetiological factors. Given the important genetic contribution to both disorders, the search for genes common to depression and migraine is a promising route for future research.

\footnotetext{
Zainab Samaan, MBChB, MRCPsych, MSC, Medical Research Council (MRC) Social Genetic and Developmental Psychiatry Centre, Institute of Psychiatry, London, UK, and Department of Behavioural Neurosciences, Mood Disorders Program, McMaster University, Hamilton, Ontario, Canada; Anne Farmer, MBChB, FRCPsych, MD, MRC Social Genetic and Developmental Psychiatry Centre, Institute of Psychiatry, London, Social Genetic and Developmental Psychiatry Centre, Institute of Psychiatry, Lond
UK; Nick Craddock, MBChB, MRCPsych, PhD, Department of Psychological Medicine, School of Medicine, Cardiff University, UK; Lisa Jones, PhD, Division of Neuroscience, University of Birmingham, UK; Ania Korszun, MD, MRCPsych, PhD, Barts and The London, Queen Mary's School of Medicine and Dentistry, London, UK: Mike Owen, MB, PhD, FRCPsych, FMedSci, Department of Psychological Medicine, Cardiff University, UK; Peter McGuffin, MB, PhD, FRCP, FRCPsych, FMedSci, MRC, Social Genetic and Developmental Psychiatry Centre, Institute of Psychiatry, London, UK

Correspondence: Dr Zainab Samaan, McMaster University, Department of Psychiatry and Behavioural Neurosciences, Mood Disorders Program, Centre for Mountain Health Services, St Joseph's Healthcare, Room D107-B, 100 West 5th Street, Box 585, Hamilton, Ontario L8N 3K7, Canada. Email: samaanz@mcmaster.ca

First received 22 Apr 2008, final revision 23 Jul 2008, accepted 23 Sep 2008
}

\section{Funding}

The study was funded by the Medical Research Council.

\section{Acknowledgements}

The authors thank Dr E. A. MacGregor, Director of Clinical Research at the City of London Migraine Clinic and Honorary Senior Clinical Lecturer at the Research Centre for Neuroscience, Queen Mary's School of Medicine and Dentistry, University of London, for her valuable help in testing the reliability of migraine interview.

\section{Appendix}

\section{Headache diagnosis criteria}

The criteria for diagnosing each category were described in the International Headache Society Manual. ${ }^{10}$ 
1. Migraine with aura: at least two episodes of aura symptoms including visual disturbances prior to the onset of headache and fulfilling criteria for migraine, as in migraine without aura below.

2. Migraine without aura: at least five episodes of moderate to severe headache with all of the following:

(a) pulsating or one-sided headache;

(b) associated with nausea and/or vomiting and/or hypersensitivity to light or sound;

(c) headache exacerbated by movement or similar physical activity;

(d) headache lasting 4-72 h.

3. Probable migraine: at least five episodes of moderate to severe headache with only three of the features listed above under 2.(a)-(d).

4. Tension type and other headaches: recurrent headaches that do not fulfil the criteria for any form of migraine or probable migraine.

5. No recurrent headache.

\section{References}

1 Merikangas KR, Stevens DE. Comorbidity of migraine and psychiatric disorders. Neurol Clin 1997; 15: 115-23.

2 Radat F, Swendsen J. Psychiatric comorbidity in migraine: a review. Cephalalgia 2005; 25: 165-78.

3 Sham PC, Sterne A, Purcell S, Cherny S, Webster M, Rijsdijk F, et al. GENESiS: creating a composite index of the vulnerability to anxiety and depression in a community-based sample of siblings. Twin Res 2000; 3: 316-22.

4 McGuffin P, Katz R, Aldrich J. Past and present state examination: the assessment of 'lifetime ever' psychopathology. Psychol Med 1986; 16: 461-5

5 Wing JK, Babor T, Brugha T, Burke J, Cooper JE, Giel R, et al. SCAN. Schedule for the Clinical Assessment of Neuropsychiatry. Arch Gen Psychiatry 1990; 47: 589-93.

6 Celik C. Computer assisted personal interviewing application for the Schedules for Clinical Assessment in Neuropsychiatry Version 2.1 and diagnostic algorithms for WHO ICD-10 chapter V DCR and for statistical manual IV. Release 1.Ed. 1.0.3.5 Win 9x NT. World Health Organization, 2003.

7 World Health Organization. International Statistical Classification of Diseases and Related Health Problems (ICD-10). WHO, 1992.

8 American Psychiatric Association. Diagnostic and Statistical Manual of Mental Disorders (4th edn) (DSM-IV). APA, 1994.

9 Beck AT, Stear RA, Garbin MG. Psychometric properties of the Beck Depression Inventory: twenty-five years of evaluation. Clin Psychol Rev 1988; 8: $77-100$

10 International Headache Society. The International Classification of Headache Disorders, 2nd edition (ICHD-II). Cephalalgia 2004; 24 (suppl 1): 8-160.

11 Breslau N, Schultz L, Stewart W, Lipton R, Lucia V, Welch K. Headache and major depression: is the association specific to migraine? Neurology 2000; 54: 308-14.

12 Merikangas KR, Angst J, Isler H. Migraine and psychopathology: results of the Zurich cohort study of young adults. Arch Gen Psychiatry 1990; 47: 849-53.

13 Merikangas KR, Merikangas JR, Angst J. Headache syndromes and psychiatric disorders: association and familial transmission. J Psychiatr Res 1993; 27: 197-210.

14 Breslau N, Davis GC. Migraine, physical health and psychiatric disorder: a prospective epidemiologic study in young adults. J Psychiatr Res 1993; 27 211-21.
15 Kendler KS, Neale MC, Kessler RC, Heath AC, Eaves L. Major depression and generalized anxiety disorder. Same genes, (partly) different environments? Arch Gen Psychiatry 1992; 49: 716-22.

16 Thapar A, McGuffin P. Anxiety and depressive symptoms in childhood a genetic study of comorbidity. J Child Psychol Psychiatry 1997; 38: 651-6.

17 Merikangas KR, Risch NJ, Merikangas JR, Weissman MM, Kidd KK. Migraine and depression: association and familial transmission. J Psychiatr Res 1988; 22: $119-29$.

18 McGuffin P, Katz R, Watkins S, Rutherford J. A hospital-based twin register of the heritability of DSM-IV unipolar depression. Arch Gen Psychiatry 1996; 53: 129-36.

19 Sullivan $\mathrm{P}$, Neale $\mathrm{M}$, Kendler KS. Genetic epidemiology of major depression: review and meta analysis. Am J Psychiatry 2000; 157: 1552-62.

20 Merikangas KR. Genetics of migraine and other headache. Curr Opin Neurol 1996; 9: 202-5.

21 Punay N, Couch J. Antidepressants in the treatment of migraine headache. Curr Pain Headache Rep 2003; 7: 51-4.

22 Rubino E, Ferrero M, Rainero I, Binello E, Vaula G, Pinessi L. Association of the C677T polymorphism in the MTHFR gene with migraine: a metaanalysis. Cephalalgia 2007 published ahead of print; doi: 10.1111/j.14682982.2007.01400.X.

23 Gilbody S, Lewis S, Lightfoot T. Methylenetetrahydrofolate reductase (MTHFR) genetic polymorphisms and psychiatric disorders: a HuGE review. Am J Epidemiol 2007; 165: 1-13.

24 Bigal ME, Liberman JN, Lipton RB. Obesity and migraine: a population study. Neurology 2006; 66: 545-50.

25 Stewart WF, Lipton RB, Celentano DD, Reed ML. Prevalence of migraine headache in the United States. Relation to age, income, race, and other sociodemographic factors. JAMA 1992; 267: 64-9.

26 Breslau N, Rasmussen BK. The impact of migraine: epidemiology, risk factors, and co-morbidities. Neurology 2001; 56 (6 suppl 1): S4-12.

27 Dalstra JAA, Kunst AE, Borrell C, Breeze E, Cambois E, Costa G, et al. Socioeconomic differences in the prevalence of common chronic diseases: an overview of eight European countries. Int J Epidemiol 2005; 34: 316-26.

28 Samaan Z, MacQueen G. Depression, migraine and cardiovascular disease: sadness really can break your heart. J Psychosom Res 2008; 65: 103-6.

29 Gudmundsson LS, Thorgeirsson G, Sigfusson N, Sigvaldason H, Johannsson M. Migraine patients have lower systolic but higher diastolic blood pressure compared with controls in a population-based study of 21537 subjects. The Reykjavik Study. Cephalalgia 2006; 26: 436-44.

30 Farmer A, Korszun A, Owen MJ, Craddock N, Jones L, Jones I, et al. Medical disorders in people with recurrent depression. Br J Psychiatry 2008; 192 $351-5$.

31 Hagen K, Stovner L, Vatten L, Holmen J, Zwart JA, Bovim G. Blood pressure and risk of headache: a prospective study of 22685 adults in Norway. J Neurol Neurosurg Psychiatry 2002; 72: 463-6.

32 Wiehe M, Costa Fuchs S, Moreira LB, Stoll Moraes R, Fuchs FD. Migraine is more frequent in individuals with optimal and normal blood pressure: a population-based study. J Hypertens 2002; 20: 1303-6.

33 D'Andrea G, welch K, Riddle J, Grunsfeld S, Joseph R. Platelet serotonin metabolism and ultrastructure in migraine. Arch Neurol 1989; 46: 1187-9.

34 Jarman J, Davies P, Fernandez M, Glover V, Steiner TJ, Rose FC, et al. Platelet $[3 \mathrm{H}] \mathrm{imipramine}$ binding in migraine and tension headache in relation to depression. J Psychiatr Res 1991; 25: 205-11.

35 Rasmussen B. Epidemiology of headache. Cephalalgia 2001; 21: 774-7.

36 Rasmussen BK, Olesen J. Migraine with aura and migraine without aura: an epidemiological study. Cephalalgia 1992; 12: 221-8. 